\title{
Myricitrin ameliorates ethanol-induced steatosis in mouse AML12 liver cells by activating AMPK, and reducing oxidative stress and expression of inflammatory cytokines
}

\author{
JING GAO $^{1-3^{*}}$, SI CHEN $^{1 *}$, ZIKAI QIU $^{4}$, LIPING FANG $^{1}$, LISHAN ZHANG $^{1}$, \\ CHANG GUO $^{1,2}$, TONG CHEN ${ }^{1,2}$ and LONGXIN QIU ${ }^{1-3}$
}

\begin{abstract}
${ }^{1}$ School of Life Sciences; ${ }^{2}$ Fujian Province Universities Key Laboratory for Preventive Veterinary Medicine and Biotechnology; ${ }^{3}$ Fujian Provincial Key Laboratory for The Prevention and Control of Animal Infectious Diseases and Biotechnology, Longyan University, Longyan, Fujian 364012; ${ }^{4}$ Light Industry and Food Engineering College, Guangxi University, Nanning 530004, P.R. China
\end{abstract}

Received August 28, 2017; Accepted February 2, 2018

DOI: $10.3892 / \mathrm{mmr} .2018 .8740$

\begin{abstract}
It is necessary to identify compounds that may provide protection against alcoholic liver disease. To the best of our knowledge, the effect of myricitrin on the development of ethanol-induced liver disease has not been previously investigated. The present study aimed to determine the effect of myricitrin on ethanol-induced steatosis in AML12 mouse liver cells and to identify the underlying molecular mechanisms. Ethanol-treated AML12 cells exhibited significantimprovement in viability following treatment with myricitrin. Oil red $\mathrm{O}$ staining indicated that myricitrin ameliorated ethanol-induced lipid accumulation in cells. Furthermore, following treatment with myricitrin, improvement in ethanol-induced steatosis and decrease in the levels of reactive oxygen species and lipoperoxides were observed in ethanol-stimulated cells. Myricitrin suppressed mRNA and protein expression of tumor necrosis factor- $\alpha$, interleukin- 6 and transforming growth factor- $\beta 1$ in ethanol-stimulated AML12 cells. Myricitrin markedly increased phosphorylation of adenosine monophosphate-activated protein kinase (AMPK) and significantly reduced mRNA expression of sterol-regulatory element-binding protein-1c (SREBP-1c) and fatty acid synthase in ethanol-stimulated AML12 cells. The results of the present study indicate that myricitrin ameliorates ethanol-induced steatosis in AML12 cells by attenuating oxidative stress,
\end{abstract}

Correspondence to: Dr Longxin Qiu, School of Life Sciences, Longyan University, 1 North Dongxiao Road, Longyan, Fujian 364012, P.R. China

E-mail: qlongxin@tom.com

*Contributed equally

Key words: myricitrin, ethanol-induced steatosis, oxidative stress, cytokines, adenosine 5'-phosphate-activated protein kinase, sterol-regulatory element-binding protein-1c suppressing expression of certain inflammatory cytokines and modulating the AMPK/SREBP-1c pathway.

\section{Introduction}

Alcoholic liver disease (ALD) is a worldwide health concern. The World Health Organization states that there are 3.3 million alcohol-associated mortalities each year (1). Excessive alcohol consumption can lead to acute and chronic fatty liver diseases, including steatosis, alcoholic hepatitis, liver fibrosis, liver cirrhosis and hepatocellular carcinoma (2). Steatosis occurs during the early stage of ALD and is characterized by excessive fat accumulation in hepatocytes (3). There are numerous mechanisms underlying fat accumulation, including de novo lipogenesis, impaired $\beta$-oxidation of fatty acids and uptake of triglyceride-rich lipoproteins (4-6). Furthermore, previous studies have demonstrated that ethanol-induced oxidative stress and associated production of cytokines have a role in the development of $\operatorname{ALD}(7,8)$.

Benign steatosis can be reversed by abstinence from alcohol; however, there are currently no effective clinical approaches for the treatment of $\operatorname{ALD}(2,3)$. Thus, it is necessary to identify compounds that may provide protection against ALD. Myricitrin (3',4',5',5,7-five hydroxyflavone-3-O- $\alpha$-L-rha mnoside), a naturally occurring polyphenol hydroxy flavonoid present in a number of berries, vegetables and various edible and/or medicinal herbs, has been reported to exhibit a variety of beneficial properties, including anti-inflammatory, antinociceptive, anticarcinogenic and antimicrobial activities (9-13). Myricitrin exhibits oxidative resistance and free radical scavenging activities due to its polyhydroxy structure (14). Myricitrin has been reported to suppress acrylamide-mediated cytotoxicity in human Caco-2 cells by inhibiting the production of reactive oxygen species (ROS) (15). Shimosaki et al (13) confirmed that pretreatment with high-performance liquid chromatography-purified myricitrin from Myrica extract can inhibit production of tumor necrosis factor- $\alpha$ (TNF- $\alpha$ ) in lipopolysaccharide-stimulated RAW264.7 macrophages. Myricitrin inhibits oxidative stress-induced endothelial 
damage and atherosclerosis $(16,17)$. It has been demonstrated that myricitrin exhibits anti-oxidative, anti-inflammatory and antifibrotic effects in carbon tetrachloride-intoxicated mice (18). Myricitrin decreased hepatic lipid peroxidation, increased levels of glutathione, reduced cyclooxygenase-2 and TNF- $\alpha$ overexpression, and inflammation in the liver, and inhibited hepatic expression of transforming growth factor- $\beta 1$ (TGF- $\beta 1$ ) and liver fibrosis (18). The above studies demonstrated anti-oxidative and anti-inflammatory activities of myricitrin under various disease conditions. However, little is known about the protective effect of myricitrin against alcoholic liver disease. The aim of the present study was to evaluate the effect of myricitrin on ethanol-induced steatosis in mouse AML12 liver cells and to identify possible underlying molecular mechanisms.

\section{Materials and methods}

Cell culture. AML12 mouse liver cells were obtained from American Type Culture Collection (Manassas, VA, USA) and cultured in Dulbecco's modified Eagle medium/nutrient mixture F12 (Hyclone; GE Healthcare Life Sciences, Logan, UT, USA) containing 100 units $/ \mathrm{ml}$ penicillin, $100 \mu \mathrm{g} / \mathrm{ml}$ streptomycin and $10 \%$ fetal bovine serum (Hyclone; GE Healthcare Life Sciences) at $37^{\circ} \mathrm{C}$ in an incubator with $5 \% \mathrm{CO}_{2}$.

Cell viability. Cell viability was assessed by an MTT assay. Briefly, AML12 cells were seeded in 96-well plates at the density of $1 \times 10^{4}$ cells/well. The following day, cells were treated with $800 \mathrm{mM}$ ethanol and co-cultured with 5, 10, 20 or $40 \mu \mathrm{M}$ myricitrin for $16 \mathrm{~h}$. Subsequently MTT solution $(5 \mathrm{mg} / \mathrm{ml})$ was added to each well and the cells were cultured for another $4 \mathrm{~h}$. MTT formazan precipitate was dissolved in dimethyl sulfoxide (DMSO) and the absorbance was measured at a wavelength of $570 \mathrm{~nm}$ using a microplate reader (Varioskan Flash; Thermo Fisher Scientific, Inc., Waltham, MA, USA). The vehicle control cells were treated with ultrapure water rather than ethanol, and DMSO rather than myricitrin. Cell viability values are expressed as percentage of the vehicle control (100\%). All experiments were performed in triplicate.

Oil red $O$ staining. AML12 cells were seeded into 24-well plates at a density of $1 \times 10^{5} /$ well. The following day, cells were stimulated with $100 \mathrm{mM}$ ethanol and co-treated with $20 \mu \mathrm{M}$ myricitrin. Following $36 \mathrm{~h}$ of treatment, cells were washed with ice-cold PBS buffer, fixed with $10 \%$ formalin at room temperature for $10 \mathrm{~min}$, and stained with oil red $\mathrm{O}$ at room temperature for $30 \mathrm{~min}$ to detect lipid droplets in cells. Following staining, the cultures were washed and the dye was extracted by isopropanol. Quantification was performed by measuring optical density at a wavelength of $510 \mathrm{~nm}$.

Detection of ROS in AML12 cells. AML12 cells were seeded in 24-well plates at a density of $1 \times 10^{5}$ cells/well. After $24 \mathrm{~h}$, cells were stimulated with $100 \mathrm{mM}$ ethanol and co-cultured with $20 \mu \mathrm{M}$ myricitrin. A total of $48 \mathrm{~h}$ after ethanol and myricitrin treatment, cells were washed and resuspended in PBS and incubated with $10 \mu \mathrm{M}$ 2',7'-dichlorofluorescin diacetate (DCF-DA) for $1 \mathrm{~h}$ at $37^{\circ} \mathrm{C}$. Subsequently, $1 \times 10^{6}$ cells $/ 200 \mu \mathrm{l} /$ well were seeded in a 96 -well microplate and DCF fluorescence was measured using a fluorescence microplate reader (Varioskan Flash; Thermo Fisher Scientific, Inc.) at an excitation wavelength of $485 \mathrm{~nm}$ and an emission wavelength of $530 \mathrm{~nm}$.

Cell lipid peroxidation assay. AML12 cells were plated and treated with ethanol and myricitrin as described above. At $48 \mathrm{~h}$ after ethanol and myricitrin treatment, total lipoperoxides were measured as thiobarbituric acid reactive substances (TBARS) in culture medium using a Lipid Peroxidation MDA Assay kit (Beyotime Institute of Biotechnology, Haimen, China), according to the manufacturer's protocol. TBARS were quantified using malondialdehyde (MDA) present in each sample.

Determination of cytokine secretion in ethanol-intoxicated cell culture. AML12 cells were plated and treated with ethanol and myricitrin as described above. A total of $48 \mathrm{~h}$ after ethanol and myricitrin treatment, the cells were centrifuged at $400 \mathrm{xg}$ at $4^{\circ} \mathrm{C}$ for $5 \mathrm{~min}$, the supernatant was collected and samples were stored at $-20^{\circ} \mathrm{C}$ until cytokine levels were measured. The concentrations of TNF- $\alpha$, interleukin (IL)- 6 and TGF- $\beta 1$ in cell supernatants were tested using the following ELISA kits: TNF- $\alpha$ (cat. no. ml002095), IL-6 (cat. no. ml002293) and TGF- $\beta 1$ (cat. no. ml002115). All kits were purchased from Shanghai Enzyme-linked Biotechnology Co., Ltd. (Shanghai, China), and performed according to the manufacturer's protocol.

Quantitative analyses of $m R N A$ expression by reverse transcription-quantitative polymerase chain reaction ( $R T-q P C R)$. Total RNA was isolated from AML12 cells using the TRIpure reagent (Roche Applied Science, Mannheim, Germany) according to the manufacturer's protocol. cDNA was synthesized from hepatic mRNA using RevertAid First Strand cDNA Synthesis kit (Thermo Fisher Scientific, Inc.). The following temperature protocol was used for cDNA synthesis: Incubation for $5 \mathrm{~min}$ at $25^{\circ} \mathrm{C}$, followed by $60 \mathrm{~min}$ at $42^{\circ} \mathrm{C}$ and then termination of the reaction via incubation for $5 \mathrm{~min}$ at $70^{\circ} \mathrm{C}$. Hepatic sterol regulatory element binding protein-1c (SREBP-1c), fatty acid synthase (FAS), TNF- $\alpha$, IL- 6 , and TGF- $\beta 1$ were analyzed using specific primers listed in Table I. qPCR was performed using FastStart Universal SYBR Green Master (Rox; Roche Applied Science). The following thermocycling conditions were used for qPCR: Initial denaturation at $95^{\circ} \mathrm{C}$ for $10 \mathrm{~min}$; 35 cycles of $95^{\circ} \mathrm{C}$ for $15 \mathrm{sec}, 53-60^{\circ} \mathrm{C}$ for $30 \mathrm{sec}$ and $72^{\circ} \mathrm{C}$ for $30 \mathrm{sec}$; and a final extension at $72^{\circ} \mathrm{C}$ for $10 \mathrm{~min}$. Relative expression of each gene was normalized to $\beta$-actin and was calculated using the comparative $2^{-\Delta \Delta \mathrm{Cq}}$ method (19).

Western blotting. Cells were homogenized in ice-cold radioimmunoprecipitation assay buffer (Beyotime Institute of Biotechnology). Total protein concentration in extracts was determined using a bicinchoninic acid protein assay kit (Beyotime Institute of Biotechnology), according to the manufacturer's protocol. Each protein sample ( $40 \mu \mathrm{g} / \mathrm{lane})$ was loaded and separated using 10\% SDS-PAGE and transferred to polyvinylidene difluoride membranes. Membranes were blocked with 5\% non-fat milk in TBS containing $0.1 \%$ Tween-20 for $1 \mathrm{~h}$ at room temperature and subsequently incubated with anti-phosphorylated (p)-adenosine 5'-phosphate-activated 
Table I. Primer sequences used for amplification of mRNA by quantitative polymerase chain reaction.

Primer sequence (5'-3')

\begin{tabular}{lll}
\cline { 2 - 3 } Gene & \multicolumn{1}{c}{ Forward } & \multicolumn{1}{c}{ Reverse } \\
\hline TNF- $\alpha$ & CGTGCTCCTCACCCACAC & GGGTTCATACCAGGGTTTGA \\
IL-6 & ACAACCACGGCCTTCCCTACTT & GTGTAATTAAGCCTCCGACT \\
TGF- $\beta 1$ & CAACTTCTGTCTGGGACCCT & TAGTAGACGATGGGCAGTGG \\
SREBP-1c & ATCGGCGCGGAAGCTGTCGGGGTAGCGTC & ACTGTCTTGGTTGTTGATGAGCTGGAGCAT \\
FAS & TGCTCCCAGCTGCAGGC & GCCCGGTAGCTCTGGGTGTA \\
$\beta$-actin & CTATTGGCAACGAGCGGTTCC & GCACTGTGTTGGCATAGAGGTC \\
\hline
\end{tabular}

TNF- $\alpha$, tumor necrosis factor- $\alpha$; IL, interleukin; TGF- $\beta 1$, transforming growth factor- $\beta 1$; SREBP-1c, sterol-regulatory element-binding protein-1c; FAS, fatty acid synthase.

protein kinase (AMPK $\alpha$; cat. no. 2535; 1:1,000 dilution), anti-AMPK $\alpha$ (cat. no. 2532; 1:1,000 dilution; both Cell Signaling Technology, Inc., Danvers, MA, USA) or GAPDH (cat. no. G9545; 1:2,000 dilution; Sigma-Aldrich; Merck $\mathrm{KGaA}$, Darmstadt, Germany) antibodies at $4^{\circ} \mathrm{C}$ overnight. Subsequently, horseradish peroxidase-conjugated anti-rabbit immunoglobulin G secondary antibody (cat. no. A6154; 1:2,000 dilution; Sigma-Aldrich; Merck KGaA) was used for an incubation at room temperature for $2 \mathrm{~h}$. Detection was performed using a Supersignal Chemiluminescent Substrate kit (Beyotime Institute of Biotechnology).

Statistical analysis. All data were processed and analyzed using GraphPad software (version 5.0; GraphPad Software, Inc., La Jolla, CA, USA) and are presented as the mean \pm standard error of the mean. One-way analysis of variance with Bonferroni's multiple comparison test were used for analyses. $\mathrm{P}<0.05$ was considered to indicate a statistically significant difference.

\section{Results}

Myricitrin exerts a protective effect against ethanol-induced cytotoxicity. The present study aimed to determine the effect of various concentrations of myricitrin $(5,10,20$ and $40 \mu \mathrm{M})$ on ethanol-induced hepatotoxicity in AML12 cells. Myricitrin alone at 5, 10, 20, or $40 \mu \mathrm{M}$ did not exert a cytotoxic effect on AML12 cells (Fig. 1A). Incubation of AML12 cells with $800 \mathrm{mM}$ ethanol for $16 \mathrm{~h}$ induced $\sim 45.5 \%$ growth inhibition compared with the untreated control $(\mathrm{P}<0.001$; Fig. 1B). Administration of 5, 10, 20, and $40 \mu \mathrm{M}$ myricitrin to ethanol-stimulated cells exerted a protective effect and reduced cell viability inhibition to $41.6(\mathrm{P}<0.01), 28.5(\mathrm{P}<0.001), 24.2$ $(\mathrm{P}<0.001)$ and $25.9 \%(\mathrm{P}<0.001)$, respectively. The above data suggest that myricitrin exerts a significant protective effect against ethanol-induced cytotoxicity. Myricitrin at a dose of $20 \mu \mathrm{M}$ exerted the most beneficial effect on ethanol-induced cytotoxicity and therefore, this dose was used in the subsequent experiments.

Myricitrin attenuates ethanol-induced steatosis in AML12 cells. Previous studies demonstrated that ethanol may induce steatosis $(20,21)$. To determine the effect of myricitrin on
A

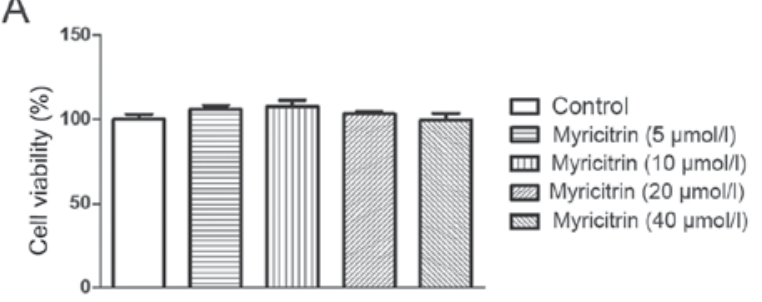

B

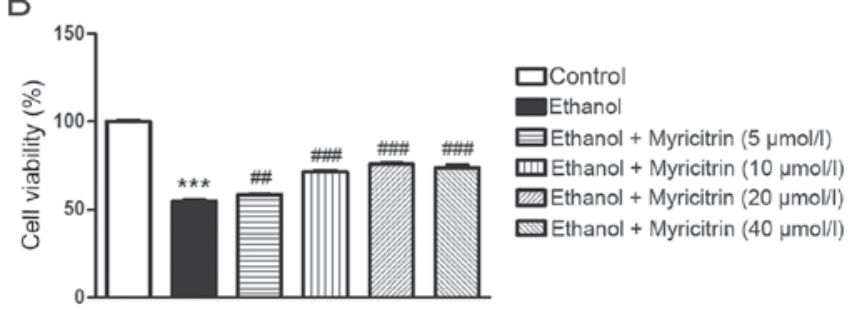

Figure 1. Myricitrin protects against ethanol-induced cytotoxicity. (A) Viability of AML12 cells treated with myricitrin. (B) Effect of myricitrin on ethanol-induced cytotoxicity. AML12 cells were treated with $800 \mathrm{mM}$ ethanol and different concentrations of myricitrin $(n=4)$. Data are presented as the mean \pm standard error of the mean. ${ }^{* * *} \mathrm{P}<0.001$ vs. the control. ${ }^{\# \#} \mathrm{P}<0.01$ and ${ }^{\# \# \#} \mathrm{P}<0.001$ vs. ethanol treated cells.

ethanol-induced steatosis, cells were treated with $100 \mathrm{mM}$ ethanol or $20 \mu \mathrm{M}$ myricitrin, or both. Oil red $\mathrm{O}$ staining demonstrated lipid accumulation in ethanol-stimulated cells while the control cells did not exhibit steatosis $(\mathrm{P}<0.001$; Fig. 2). Following co-culture with myricitrin, lipid accumulation in ethanol-stimulated cells was significantly attenuated $(\mathrm{P}<0.001)$. The results indicate that myricitrin can suppress the development of ethanol-induced steatosis in hepatocytes.

Myricitrin reduces ethanol-induced oxidative stress and decreases mRNA expression of certain cytokines in AML12 cells. Oxidative stress is reported to be involved in the development of ethanol-induced steatosis (2). The present study investigated the effect of myricitrin treatment on MDA and ROS production in AML12 cells. Treatment with myricitrin resulted in a $32.1 \%$ decrease in ROS levels $(\mathrm{P}<0.001)$ and $22.4 \%$ decrease in MDA levels $(\mathrm{P}<0.001)$ in ethanol-stimulated AML12 cells (Fig. 3). The above data indicate that myricitrin 
A

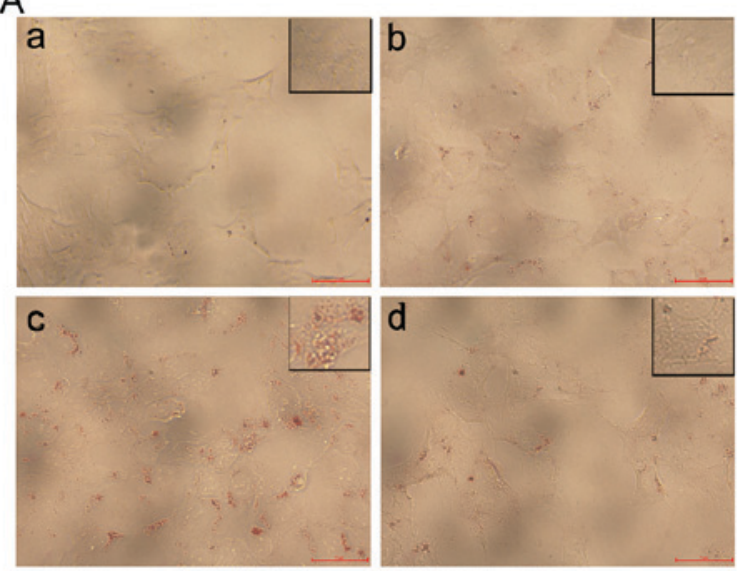

B

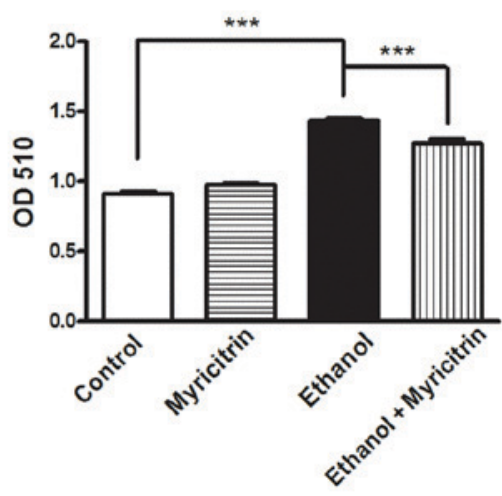

Figure 2. Myricitrin treatment ameliorates ethanol-induced steatosis in AML12 cells. (A) Oil red O staining of cells in (A-a) control group without ethanol stimulation, (A-b) myricitrin group, (A-c) ethanol-stimulated cells and (A-d) ethanol + myricitrin group. Images are representative of six separate experiments (original magnification of images a-d, x400; and magnification of the magnified sections within these images, x800). (B) Cell lipid accumulation was quantified by extracting the dye with isopropanol and measuring its absorbance at a wavelength of $510 \mathrm{~nm}(\mathrm{n}=6)$. Data are presented as the mean \pm standard error of the mean. ${ }^{* * *} \mathrm{P}<0.001$.

alleviates ethanol-induced oxidative stress in liver cells. Certain pro-inflammatory cytokines, including TNF- $\alpha$, IL- 6 and TGF- $\beta 1$ have been reported to be associated with the development of ethanol-induced hepatic steatosis $(8,22)$. Expression levels of TNF- $\alpha$, IL- 6 and TGF- $\beta 1$ were also been determined in the present study. Cells stimulated with ethanol exhibited markedly elevated mRNA expression levels of TNF- $\alpha$, compared with control cells (Fig. 4A). However, ethanol-induced increase in TNF- $\alpha$ mRNA levels was significantly attenuated in cells treated with myricitrin $(47.9 \%$; $\mathrm{P}<0.001)$. Similarly, cells stimulated with ethanol exhibited markedly increased expression of IL- 6 and TGF- $\beta 1$ mRNA compared with control cells. This ethanol-induced elevation of mRNA levels of IL- 6 and TGF- $\beta 1$ was significantly attenuated, by $27.1 \%(\mathrm{P}<0.01)$ and $51.8 \%(\mathrm{P}<0.001)$ respectively, in cells treated with myricitrin. Furthermore, ethanol-induced secretion of TNF- $\alpha$, IL- 6 and TGF- $\beta 1$ proteins was also suppressed by $28.3 \%(\mathrm{P}<0.01), 44.6 \%(\mathrm{P}<0.05)$ and $43.8 \%$ $(\mathrm{P}<0.01)$, respectively, in cells treated with myricitrin (Fig. 4B). The results suggest that myricitrin may reduce oxidative stress and affect the production of pro-inflammatory cytokines to prevent ethanol-induced steatosis in AML12 liver cells.

Myricitrin activates AMPK and reduces the ethanol-induced elevation of SREBP-1c and FAS mRNA expression in AML12 cells. Previous studies indicated that AMPK inactivation may be involved in the development of ethanol-induced hepatic steatosis $(23,24)$. To determine the mechanism underlying myricitrin-induced amelioration of ethanol-induced steatosis, the present study investigated the effect of treatment with myricitrin on AMPK activity. pAMPK protein expression levels in ethanol-stimulated AML12 cells treated with myricitrin increased compared with myricitrin untreated ethanol-stimulated cells, indicating that myricitrin treatment activated AMPK in ethanol-stimulated AML12 cells (Fig. 5A). Furthermore, the effect of treatment with myricitrin on mRNA expression of SREBP-1c and FAS was determined. SREBP-1c and FAS genes are regulated by AMPK and control the synthesis of fatty acids in ethanol-stimulated liver cells $(21,25,26)$. Treatment with $100 \mathrm{mM}$ ethanol resulted in a significant increase in SREBP-1c mRNA expression levels compared with the control group and this increase was significantly attenuated following treatment of ethanol-stimulated cells with myricitrin $(\mathrm{P}<0.05$; Fig. 5B). mRNA expression of FAS significantly decreased in myricitrin and ethanol-stimulated cells compared with the ethanol treatment group $(\mathrm{P}<0.01$; Fig. 5C). The above results indicate that treatment with myricitrin may ameliorate ethanol-induced steatosis in AML12 cells through activation of AMPK and subsequent inhibition of SREBP-1c-regulated lipogenesis.

\section{Discussion}

Previous studies have demonstrated that myricitrin may protect against liver injury by attenuating oxidative stress in $\mathrm{CCl}_{4}$-intoxicated mice (18). In the present study, myricitrin suppressed ethanol-induced production of MDA and ROS in mouse AML12 liver cells. The results of the present study further indicate that myricitrin may attenuate oxidative stress in chemically intoxicated cells. Oxidative stress has been hypothesized to serve a role in pathogenesis of ALD (2). The results of the present study suggest that myricitrin may protect against ALD by attenuating oxidative stress.

The incidence and development of alcoholic liver disease are associated with overexpression of a number of cytokines, including TNF- $\alpha$, IL- 6 and TGF- $\beta 1(8,27,28)$. Previous studies have demonstrated that expression of the inflammatory cytokine TNF- $\alpha$ is elevated in patients with alcoholic liver disease $(8,29)$. TNF- $\alpha$ has been reported to induce hepatic steatosis in mice by affecting hepatic lipogenic metabolism (30). Other studies have indicated that expression of IL-6 is elevated in patients with alcoholic liver diseases $(27,28)$. Persistent activation of IL-6 signaling may be detrimental to the liver and may cause liver tumors (31). The present study demonstrated that myricitrin may reduce mRNA expression of TNF- $\alpha$, IL- 6 and TGF- $\beta 1$, indicating that alleviation of 
A

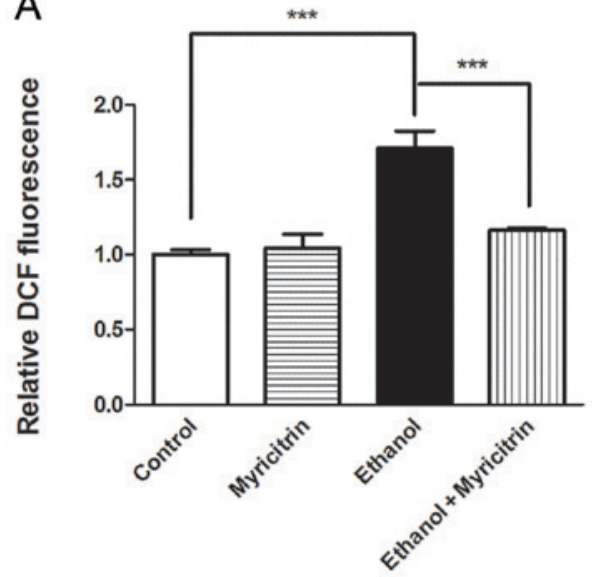

B

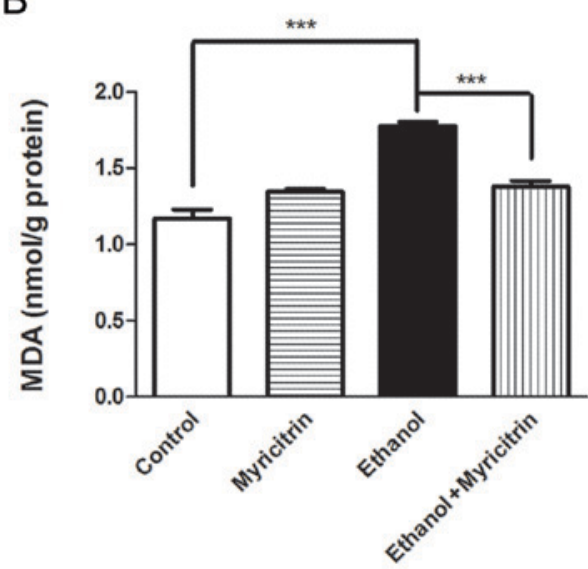

Figure 3. Myricitrin decreases levels of ROS and MDA in AML12 cells. (A) ROS and (B) MDA levels were assayed in ethanol-stimulated AML12 cells treated with myricitrin for $36 \mathrm{~h}(\mathrm{n}=4)$. Data are presented as the mean \pm standard error of the mean. ${ }^{* * *} \mathrm{P}<0.001$. DCF, dichlorofluorescin; ROS, reactive oxygen species; MDA, malondialdehyde.
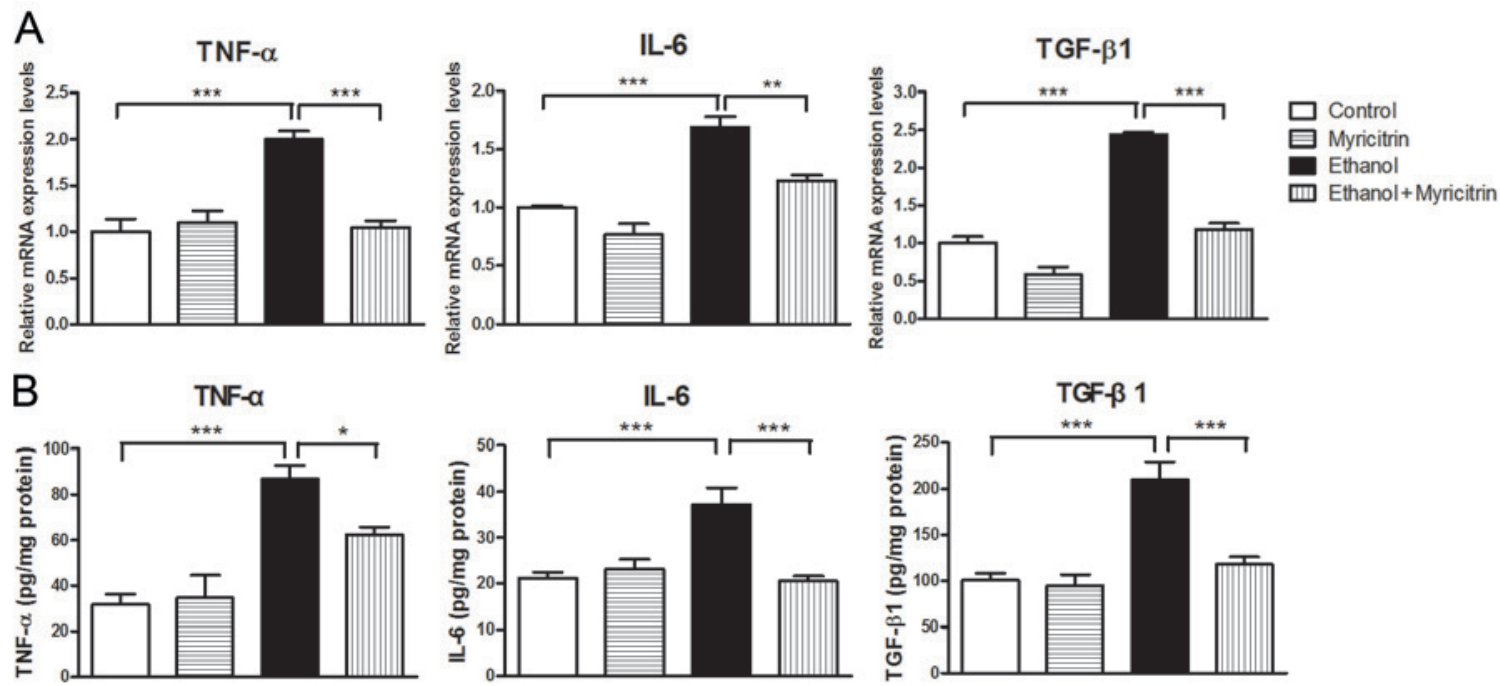

Figure 4. Myricitrin attenuates mRNA expression and protein secretion of cytokines in ethanol-stimulated AML12 cells. (A) mRNA expression levels of TNF- $\alpha$, IL- 6 and TGF- $\beta 1$ were analyzed by reverse transcription-quantitative polymerase chain reaction, standardized using $\beta$-actin internal control and expressed as fold differences relative to values observed in control cells $(n=3)$. (B) Cytokines TNF- $\alpha$, IL- 6 and TGF- $\beta 1$ secreted into cell culture media were analyzed by ELISA assays, and standardized against protein contents of cells. Data are presented as the mean \pm standard error of the mean. ${ }^{*} \mathrm{P}<0.05 ;{ }^{* *} \mathrm{P}<0.01$; ${ }_{* * * *} \mathrm{P}<0.001$. TNF- $\alpha$, tumor necrosis factor- $\alpha$; IL, interleukin; TGF- $\beta 1$, transforming growth factor- $\beta 1$.

A

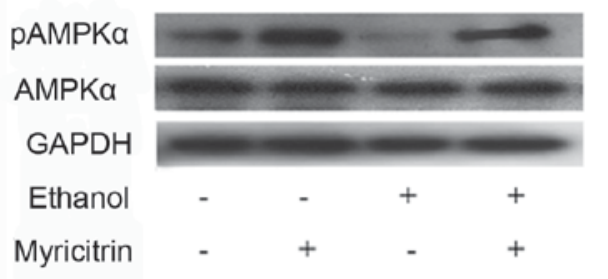

B

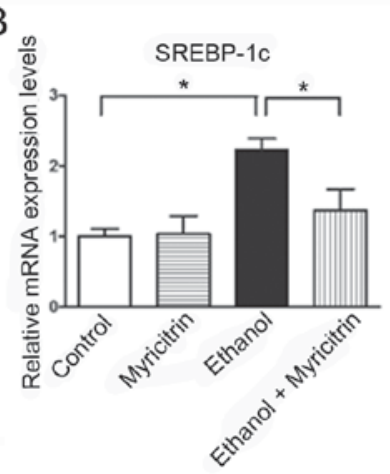

C

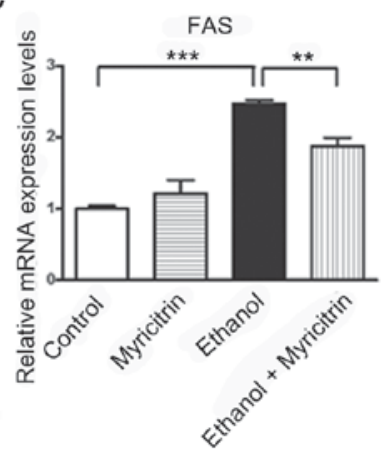

Figure 5. Effect of treatment with myricitrin on activation of AMPK and mRNA expression of SREBP-1c and FAS. (A) Activation of AMPK in myricitrin-treated AML12 liver cells stimulated with ethanol was determined by western blotting. mRNA expression of (B) SREBP-1c and (C) FAS in myricitrin-treated AML12 liver cells stimulated with ethanol was analyzed by reverse transcription-quantitative polymerase chain reaction standardized using $\beta$-actin internal control and expressed as fold differences relative to values observed in control cells $(\mathrm{n}=3)$. Data are presented as the mean \pm standard error of the mean. ${ }^{*} \mathrm{P}<0.05 ;{ }^{* * *} \mathrm{P}<0.01$; ${ }^{* * * *} \mathrm{P}<0.001$. AMPK, adenosine monophosphate-activated protein kinase; SREBP-1c, sterol-regulatory element-binding protein-1c; FAS, fatty acid synthase. 
ethanol-induced steatosis in liver cells caused by myricitrin may be partially due to inhibition of ethanol-induced overexpression of TNF- $\alpha$, IL- 6 and TGF- $\beta 1$ mRNA.

AMPK has a role in cellular energy homeostasis (32). Inhibition of AMPK suppresses fatty acid oxidation and stimulates lipogenesis (33). Ethanol has been reported to inhibit AMPK activity in vitro (24) and in vivo (34). Inhibition of AMPK by ethanol has a role in the development of hepatosteatosis induced by alcohol consumption $(23,24)$. SREBP-1c is a regulator of hepatic lipid metabolism and is involved transcription of a number of genes involved in liver triglyceride and fatty acid synthesis, such as FAS (35-37). Increased expression of SREBP-1c induced by ethanol may cause hepatic lipid accumulation in alcoholic fatty liver (24). AMPK activators have been demonstrated to reduce the expression of SREBP-1c (24). In the present study, myricitrin activated AMPK in ethanol-stimulated AML12 liver cells. The results also demonstrated that myricitrin suppressed mRNA expression of SREBP-1c and FAS. Suppression of SREBP-1c expression and activation of AMPK were observed in ethanol-induced cells following treatment with myricitrin. The results of the present study indicate that myricitrin regulates the AMPK/SREBP-1c signaling pathway and reduces lipid formation in liver cells. However, specific inhibitors or gene specific-short hairpin RNAs may be used in the future to alter the activity of AMPK following treatment with myricitrin to further validate the specificity of myricitrin to the AMPK/SREBP signaling pathway.

Aldose reductase (AKR1B1) is an enzyme that catalyzes the reduction of glucose to sorbitol with the aid of co-factor NADPH in the polyol pathway (38). We previously reported that AKR1B1 inhibitor ameliorates ethanol-induced steatosis in vitro and in vivo by activating AMPK and suppressing expression of SREBP-1c and FAS $(21,25)$. Myricitrin is an inhibitor of AKR1B1 $(39,40)$. In the present study, myricitrin ameliorated ethanol-induced steatosis via activation of AMPK and suppression of expression of SREBP-1c and FAS, thus indicating that the beneficial effect of myricitrin on ethanol-induced steatosis may be at least partially attributable to inhibition of AKR1B1.

In conclusion, myricitrin ameliorated ethanol-induced lipid abnormalities in AML12 liver cells, which was partially due to suppression of ethanol-induced oxidative stress and associated with suppression of ethanol-induced overexpression of certain cytokines. The present study also demonstrated that myricitrin activated AMPK and suppressed SREBP-1c and FAS mRNA overexpression to alleviate the development of hepatic steatosis. The present study may provide theoretical basis for therapeutic use of AKR1B1 inhibitor in the treatment of fatty liver disease.

\section{Acknowledgements}

The authors would like to thank Mrs. Ailing Dai and Mrs. Chunhua Wei of Longyan University for their technical support.

\section{Funding}

The present study was supported in part by Natural Science Foundation of Fujian Province, China (grant no. 2015J01619) and by Fujian Provincial Undergraduate Training Programs for Innovation and Entrepreneurship, China (grant. no. 201611312026).

\section{Availability of data and materials}

The datasets used and/or analyzed during the current study are available from the corresponding author on reasonable request.

\section{Authors' contributions}

LQ conceived and designed the experiments, and wrote the manuscript; JG, SC, ZQ, LF and LZ performed the experiments and analyzed the data; LQ, CG and TC contributed reagents/materials/analysis tools and analyzed the data.

\section{Ethics approval and consent to participate}

Not applicable.

\section{Consent for publication}

Not applicable.

\section{Competing interests}

The authors declare that they have no competing interests.

\section{References}

1. Chacko KR and Reinus J: Spectrum of alcoholic liver disease. Clin Liver Dis 20: 419-427, 2016.

2. Louvet A and Mathurin P: Alcoholic liver disease: Mechanisms of injury and targeted treatment. Nat Rev Gastroenterol Hepatol 12: 231-242, 2015.

3. Osna NA, Donohue TM Jr and Kharbanda KK: Alcoholic liver disease: Pathogenesis and current management. Alcohol Res 38: 147-161, 2017.

4. Sun X, Tang Y, Tan X, Li Q, Zhong W, Sun X, Jia W, McClain CJ and Zhou Z: Activation of peroxisome proliferator-activated receptor-gamma by rosiglitazone improves lipid homeostasis at the adipose tissue-liver axis in ethanol-fed mice. Am J Physiol Gastrointest Liver Physiol 302: G548-G557, 2012.

5. Ji C, Chan C and Kaplowitz N: Predominant role of sterol response element binding proteins (SREBP) lipogenic pathways in hepatic steatosis in the murine intragastric ethanol feeding model. J Hepatol 45: 717-724, 2006.

6. Wang Z, Dou X, Li S, Zhang X, Sun X, Zhou Z and Song Z: Nuclear factor (erythroid-derived 2)-like 2 activation-induced hepatic very-low-density lipoprotein receptor overexpression in response to oxidative stress contributes to alcoholic liver disease in mice. Hepatology 59: 1381-1392, 2014.

7. Ishii $\mathrm{H}$, Kurose I and Kato S: Pathogenesis of alcoholic liver disease with particular emphasis on oxidative stress. J Gastroenterol Hepatol 12: S272-S282, 1997.

8. Kawaratani H, Tsujimoto T, Douhara A, Takaya H, Moriya K, Namisaki T, Noguchi R, Yoshiji H, Fujimoto M and Fukui H: The effect of inflammatory cytokines in alcoholic liver disease. Mediators Inflamm: 495156, 2013.

9. Cushnie TP and Lamb AJ: Antimicrobial activity of flavonoids. Int J Antimicrob Agents 26: 343-356, 2005

10. Kale A, Gawande S and Kotwal S: Cancer phytotherapeutics: Role for flavonoids at the cellular level. Phytother Res 22: 567-577, 2008.

11. Meotti FC, Fachinetto R, Maffi LC, Missau FC, Pizzolatti MG, Rocha JB and Santos AR: Antinociceptive action of myricitrin: Involvement of the $\mathrm{K}+$ and $\mathrm{Ca} 2+$ channels. Eur J Pharmacol 567: 198-205, 2007. 
12. Qi S, Feng Z, Li Q, Qi Z and Zhang Y: Myricitrin modulates NADPH oxidase-dependent ROS production to inhibit endotoxin-mediated inflammation by blocking the JAK/STAT1 and NOX2/p47phox pathways. Oxid Med Cell Longev 20147: 9738745, 2017.

13. Shimosaki S, Tsurunaga $\mathrm{Y}$, Itamura $\mathrm{H}$ and Nakamura $\mathrm{M}$ : Anti-allergic effect of the flavonoid myricitrin from Myrica rubra leaf extracts in vitro and in vivo. Nat Prod Res 25: 374-380, 2011

14. Wu JH, Huang CY, Tung YT and Chang ST: Online RP-HPLC-DPPH screening method for detection of radical-scavenging phytochemicals from flowers of Acacia confusa. J Agric Food Chem 56: 328-332, 2008.

15. Chen W, Feng L, Shen Y, Su H, Li Y, Zhuang J, Zhang L and Zheng X: Myricitrin inhibits acrylamide-mediated cytotoxicity in human Caco-2 cells by preventing oxidative stress. Biomed Res Int: 724183, 2013.

16. Sun GB, Qin M, Ye JX, Pan RL, Meng XB, Wang M, Luo Y, Li ZY, Wang HW and Sun XB: Inhibitory effects of myricitrin on oxidative stress-induced endothelial damage and early atherosclerosis in ApoE-/- mice. Toxicol Appl Pharmacol 271: 114-126, 2013.

17. Qin M, Luo Y, Meng XB, Wang M, Wang HW, Song SY, Ye JX Pan RL, Yao F, Wu P, et al: Myricitrin attenuates endothelial cell apoptosis to prevent atherosclerosis: An insight into PI3K/Ak activation and STAT3 signaling pathways. Vascul Pharmacol 70 : 23-34, 2015.

18. Domitrović R, Rashed K, Cvijanović O, Vladimir-Knežević S, Škoda M and Višnić A: Myricitrin exhibits antioxidant, anti-inflammatory and antifibrotic activity in carbon tetrachloride-intoxicated mice. Chem Biol Interact 230: 21-29, 2015.

19. Livak KJ and Schmittgen TD: Analysis of relative gene expression data using real-time quantitative PCR and the 2(-Delta Delta C(T)) Method. Methods 25: 402-408, 2001.

20. Altamirano $J$ and Bataller R: Alcoholic liver disease: Pathogenesis and new targets for therapy. Nat Rev Gastroenterol Hepatol 8: 491-501, 2011

21. Qiu L, Cai C, Zhao X, Fang Y, Tang W and Guo C: Inhibition of aldose reductase ameliorates ethanol induced steatosis in HepG2 cells. Mol Med Rep 15: 2732-2736, 2017.

22. Thiele DL: Tumor necrosis factor, the acute phase response and the pathogenesis of alcoholic liver disease. Hepatology 9: 497-499, 1989.

23. Sid B, Verrax J and Calderon PB: Role of AMPK activation in oxidative cell damage: Implications for alcohol-induced liver disease. Biochem Pharmacol 86: 200-209, 2013

24. You M, Matsumoto M, Pacold CM, Cho WK and Crabb DW: The role of AMP-activated protein kinase in the action of ethanol in the liver. Gastroenterology 127: 1798-1808, 2004.

25. Shi C, Wang Y, Gao J, Chen S, Zhao X, Cai C, Guo C and Qiu L: Inhibition of aldose reductase ameliorates alcoholic liver disease by activating AMPK and modulating oxidative stress and inflammatory cytokines. Mol Med Rep 16: 2767-2772, 2017.
26. Hong SH, Lee H, Lee HJ, Kim B, Nam MH, Shim BS and Kim SH: Ethanol extract of pinus koraiensis leaf ameliorates alcoholic fatty liver via the activation of LKB1-AMPK signaling in vitro and in vivo. Phytother Res 31: 783-791, 2017.

27. Neuman MG, Maor Y, Nanau RM, Melzer E, Mell H, Opris M, Cohen L and Malnick S: Alcoholic liver disease: Role of cytokines. Biomolecules 5: 2023-2034, 2015.

28. Prystupa A, Kiciński P, Sak J, Boguszewska-Czubara A, Torun-Jurkowska A and Zaluska W: Proinflammatory cytokines (IL-1alpha, IL-6) and hepatocyte growth factor in patients with alcoholic liver cirrhosis. Gastroenterol Res Pract 2015: 532615, 2015.

29. McClain CJ and Cohen DA: Increased tumor necrosis factor production by monocytes in alcoholic hepatitis. Hepatology 9: 349-351, 1989.

30. Endo M, Masaki T, Seike M and Yoshimatsu H: TNF-alpha induces hepatic steatosis in mice by enhancing gene expression of sterol regulatory element binding protein-1c (SREBP-1c). Exp Biol Med (Maywood) 232: 614-621, 2007.

31. Schmidt-Arras D and Rose-John S: IL-6 pathway in the liver: From physiopathology to therapy. J Hepatol 64: 1403-1415, 2016.

32. Garcia D and Shaw RJ: AMPK: Mechanisms of cellular energy sensing and restoration of metabolic balance. Mol Cell 66: 789-800, 2017.

33. Viollet B, Guigas B, Leclerc J, Hébrard S, Lantier L, Mounier R, Andreelli $\mathrm{F}$ and Foretz M: AMP-activated protein kinase in the regulation of hepatic energy metabolism: From physiology to therapeutic perspectives. Acta Physiol (Oxf) 196: 81-98, 2009.

34. García-Villafranca J, Guillén A and Castro J: Ethanol consumption impairs regulation of fatty acid metabolism by decreasing the activity of AMP-activated protein kinase in rat liver. Biochimie 90: 460-466, 2008.

35. Ferré $\mathrm{P}$ and Foufelle F: SREBP-1c transcription factor and lipid homeostasis: Clinical perspective. Horm Res 68: 72-82, 2007.

36. Ferré P and Foufelle F: Hepatic steatosis: A role for de novo lipogenesis and the transcription factor SREBP-1c. Diabetes Obes Metab 12 (Suppl 2): 83-92, 2010

37. Horton JD, Goldstein JL and Brown MS: SREBPs: Activators of the complete program of cholesterol and fatty acid synthesis in the liver. J Clin Invest 109: 1125-1131, 2002.

38. Hers HG: [Aldose reductase]. Biochim Biophys Acta 37: 120-126, 1960.

39. Varma SD, Mikuni I and Kinoshita JH: Flavonoids as inhibitors of lens aldose reductase. Science 188: 1215-1216, 1975.

40. Veeresham C, Rama Rao A and Asres K: Aldose reductase inhibitors of plant origin. Phytother Res 28: 317-333, 2014. 